\title{
Paläoklimatische Auswertung von Bündner Naturchroniken
}

\section{Einleitung}

Den vielfältigen Naturphänomenen Graubündens wurde schon früh von sehr verschiedenen Beobachtern Beachtung geschenkt. Es waren dies u. a. Durchreisende aus aller Herren Ländern, die in ihren Reiseaufzeichnungen und Landschaftsporträts die unterschiedlichsten Erscheinungen festhielten. Dies mag aber nicht sonderlich erstaunen, wenn man bedenkt, daß der Durchgangsverkehr im rätischen Alpengebiet auf Grund der zahlreichen, teilweise noch gut erhaltenen Reste römischer und mittelalterlicher Straßenzüge längs der Pässe sehr bedeutend gewesen sein muß. Die in St. Moritz gefundene bronzezeitliche Quellfassung des dortigen Sauerbrunnens bezeugt einen schon vor 3000 Jahren erfolgten Besuch von «Kurgästen». S. MARGADANT (1978) hat in seiner Dissertation "Graubünden im Spiegel der Reiseberichte und der landeskundlichen Literatur des 16.-18. Jahrhunderts" solche Dokumente der Reiseliteratur von 1492-1800 gesammelt und kommentiert. Was die alten Landschaftsansichten reisender Künstler betrifft, besitzen wir heute eine sehr wertvolle Kompilation von B. WEBER (1984), der Ansichten Graubündens aus dem 16. bis zum frühen 19. Jahrhundert sammelte und erläuterte.

Das mehrheitlich qualitative, aber reichlich vorhandene Informationsmaterial kann in fünf Gruppen eingeteilt werden:

1. Beobachtungen von einheimischen Intellektuellen, wie z.B. U.CAMPELL, der «Vater der rätischen Geschichtsschreibung» (1584, editiert von C.J.KIND, 1884), H.ARDÜSER (1572-1614), N.SERERHARD (1742), Pater P. a SPESCHA (1818), J. R. VON SALIS-MARSCHLINS (1804/1805), G. THEOBALD (1862-1893), CHR. BRÜGGER $(1882 / 1888)$ und A. VON FLUGI $(1919 / 1920)$.

2. Alte Chroniken, wie diejenigen von JOH.GULER vON WYNECK (1616), FORT.SPRECHER VON BERNECK (1672) und C. VON MOOR (1853/1876).

3. Naturchroniken (d. h. Aufzeichnungen von Naturerscheinungen verschiedenster Art, wie Unwetter, Messungen von Wetterelementen, astronomische Erscheinungen, Gletscherbewegungen, Obst-, Ge- treide-, Weinerträge, Blühbeginn verschiedener Pflanzen). Hierzu finden sich Angaben z.B. in den Zeitschriften «Der Sammler» (1779-1784), «Der neue Sammler» (1804-1812) und "Alpina» (1806-1809). H. ARDÜSER, P.SPESCHA, E.KILLIAS $(1869,1883,1890)$, W. WASSALI (1859), A.VON FLUGI (1919/1920), F.VON SALIS-MARSCHLINS $(1883 / 1887)$ und vor allem CHR. BRÜGGER (1882/1888) lieferten Beiträge zur BündnerNaturchronik, die teilweise im zweiten Kapitel ausgewertet werden.

4. Berichte von Forschungsreisenden. Hierzu seien die Aufzeichnungen von G.B. BURGO (1689), J. J. SCHEUCHZER (1705-1708), B. HACQUET (1785), H.L.LEHMANN $(1790,1798,1797 / 99)$, H.ZSCHOKKE (1797/1798), H.C.ESCHER VON DER LINTH (ed. G.SOLAR, 1974), J.G.EBEL (1809-1810), F.B.DE MERCEY (1833) und K. KASTHOFER (1822) erwähnt.

5. Briefe und Berichte der übrigen Reisenden. Diese Gruppe ist erwartungsgemäß die größte und ist in der Qualität der objektiven Berichterstattung sehr heterogen. Es handelt sich meistens um Reisende der oberen Schichten, die vor allem im 17., 18. und 19. Jahrhundert «Bildungsreisen» unternahmen. Als Beispiele seien lediglich erwähnt G.BURNET (1686, 1687), w.COXE (1792), H.ZSCHOKKE (1797), J.G.EBEL (1809), C.M.ENGELHARDT (1840). Über illustre Reisende durch Rätien, wie J.W.GOETHE, F.NIETZSCHE, T. MANN, T. FONTANE, J. V. VON SCHEFFEL u. a. berichteten P.E. MÜLLER (1972) und H. RIBI (1973). Vor allem im 18. und 19. Jahrhundert wurden mehrere Reisehandbücher oder ähnliche Publikationen verfaßt, wie z.B. die berühmte "Anleitung» J. G. EBELS (1804/1805). Weitere bedeutende Reisehandbücher bzw. -abbildungswerke stammen u.a. von J.LANGE (1852), E. PINGRET (1872), A. VON SCHADEN (1834), R. TÖPFFER (1844), P.C.VON TSCHARNER (1829/1831), G. VON BAVIER (1835).

Conradin A. Burga, Dr. phil. nat.

Geographisches Institut der Universität Zürich-Irchel,

Winterthurerstr. 190, 8057 Zürich 


\section{A usw ertungen}

Die Aufzeichnungen aus der Zeit vor den regelmäßigen Instrumenten-Beobachtungen, d.h. vor dem Höhepunkt der «Kleinen Eiszeit» (H. VON RUDLOFF, 1967), sind, wie oben bereits erwähnt, hauptsächlich qualitativer Natur. Über allgemeine Gesichtspunkte der Möglichkeiten und Grenzen der Auswertung von Quellenmaterial berichtete bereits C.PFISTER 1977 und vorwiegend 1984 in extenso, weshalb dieser Hinweis genügen möge.

Die ausführlichste und zeitlich umfassendste Naturchronik Graubündens erstellte CHR.BRÜGGER, veröffentlicht 1882/1888 im Programm der Bündnerischen Kantonsschule Chur (I.-V.Folge: 1882, VI. Folge: 1888), für die Jahre 1043-1800. Eine gewisse Fortsetzung bilden die Aufzeichnungen für die Jahre 1867-1920, die in den Jahresberichten der Naturforschenden Gesellschaft Graubünden veröffentlicht wurden. Unter den Autoren ist hierbei vor allem E.KILLIAS zu nennen mit für Graubünden und allgemein wertvollen Beobachtungen, die 1869, 1883 und $1890 \mathrm{im}$ oben genannten Periodikum publiziert wurden.

Für die Jahre 1850-1900 sammelte A. VON FLUGI (1919/1920) klimatologische Daten und Naturereignisse aus dem Oberengadin und seiner Umgebung. In der berühmten Chronik von 1572-1614 des Bündner Lehrers und Malers H. ARDÜSER finden sich auch einige klimatologische Hinweise, die weiter unten aufgeführt werden.

Aus den von F. WASSALI (1859) gesammelten Beobachtungen zum Weinbau in Graubünden lassen sich wertvolle indirekte Hinweise zum damaligen Klima gewinnen.

Einige qualitative Angaben zum Klima des 18. und 19. Jahrhunderts lassen sich aus den Aufzeichnungen der Jahre 1783-1810 von J.R. VON SALIS-MARSCHLINS herauslesen. Seine umfangreichen Messungen von Luftdruck und Temperatur (vgl. Kap. 2.5) sind wegen der damaligen Geräte-Ungenauigkeiten leider kaum brauchbar.

Schließlich seien noch die Aufzeichnungen von CATANI und ENGEL (1805) erwähnt, die lawinenreiche Jahre und die Todesopfer beinhalten.

\subsection{Die Naturchronik für die Jahre 1043-1800 von Chr.Brügger}

CHR. BRÜGGER (1833-1899) aus Churwalden studierte in Innsbruck Botanik. 1859 wurde er von O. HEER als Konservator an das Botanische Museum der ETH in Zürich berufen. Nach dem Tode G.L.THEOBALDS wurde CHR. BR ÜGGER ab 1870 sein Nachfolger für die Fächer Naturkunde und Geographie an der Bündner Kantonsschule, wo er bis zu seinem Tode wirkte. Kennzeichnend für BRÜGGER waren seine sehr weit gespannten naturwissenschaftlichen und historischen
Interessen, zu denen auch die Klimatologie und Meteorologie gehörten. Er installierte in Graubünden etwa 20 neue Stationen, die später von der Schweizerischen Meteorologischen Anstalt teilweise übernommen wurden (H. BRUNNER, 1970).

Die klimatologischen bzw. phänologischen Angaben sind alle qualitativ. Die Naturchronik wurde nach folgenden Aussagen durchgemustert: "Sehr kalter/strenger Winter", "strenge Winter und kühle, nasse Sommer», "Zürichseegfrörni», "Bodenseegfrörni», weitere gefrorene Seen: Genfer-, Vierwaldstätter-, Neuenburgersee, "kältester und schneereichster Winter des Jahrhunderts», «lawinenreiche Winter», "Gletscherausbruch», "unfruchtbare Jahre», "Weinreben erfroren", «Überschwemmungen", "Hochwasser». In der nachfolgenden tabellarischen Auswertung bedeuten die unterstrichenen Jahrgänge besonders exzessive klimatische Ereignisse (Tabellen la und $1 b$ ).

\subsection{Aus der Naturchronik für die Jahre 1867, 1880 und 1888 von E. Killias}

Für paläoklimatische Belange sind folgende Naturereignisse hervorzuheben:

Winter 1866/1867: Zahlreiche Lawinen, die große Schäden anrichteten.

Starker Schneefall, wie in den Jahren 1826, 1837, 1863.

A.D. 1867: Fröste und viele Rüfen; Felssturz von Felsberg am 12./13. Mai

A.D. 1879/1880: Anhaltende Winterkälte im Dezember und Januar; kältester Winter seit 1829/1830. Zürichsee ganz gefroren, Bodensee teilweise gefroren. Frostschäden an Efeu und Wacholder. Obst- und Zierbäume mit gesprungener Rinde, Schäden an Weinreben.

Winter 1887/1888: Ungewöhnlich schneereich (1-3 m) und viele Lawinen, besonders im Februar, März und vereinzelt im Mai.

A.D. 1888: Im September anhaltender Regen mit Hochwasser, das Straßen und Brücken zerstörte.

Der Poschiavino verursachte Überschwemmungen, besonders in Brusio wurden Straßen und Brücken zerstört. Ein Hagelwetter zerstörte die Ernte an Korn, Tabak und Reben. Das Jahr 1888 wird als Unglücksjahr bezeichnet.

\subsection{Aus der Chronik von H. Ardüser (1572-1614)}

H. ARDÜSER (1557-1618) von Davos war im Hauptberuf Schulmeister in Thusis, Savognin, Scharans und Lenz. Im Sommer bereiste er ganz Graubünden, um da und dort Malaufträge auszuführen. Gleichzeitig schrieb er an seiner kulturhistorisch interessanten Wanderchronik (C. SIMONETT, 1970). 
Tabelle 1a

\begin{tabular}{|c|c|c|c|c|}
\hline Aussage & 11. und 12. Jh. & 13. und 14. Jh. & 15. Jh. & 16. Jh. \\
\hline Sehr kalter/strenger Winter & $\begin{array}{l}\text { A.D. } 1043,1044, \\
1057,1063,1076, \\
1088,1124,1150\end{array}$ & $\begin{array}{l}\text { A.D. } 1210,1225,1228 \text {, } \\
1233,1234,1276 \text {, } \\
\underline{1363}, 1372\end{array}$ & - & - \\
\hline $\begin{array}{l}\text { Strenge Winter und kühle, } \\
\text { nasse Sommer }\end{array}$ & - & - & $\begin{array}{l}\text { A.D. } 1407, \underline{1435}, 1438 \text {, } \\
\underline{1443}, 1456,1459 \\
1464, \underline{1491}, \underline{1492}\end{array}$ & $\begin{array}{l}\text { A.D. } 1502,1506,1508, \\
1511, \underline{1514}, 1515, \\
1559 / 60, \underline{1563}, 1564 \\
1566, \underline{1571}, \underline{1573}, \underline{1585}, \\
1587,1594, \underline{1598}\end{array}$ \\
\hline Zürichsee-Gfrörni & - & A.D. $\underline{1363}$ & $\begin{array}{l}\text { A.D. } 1407, \underline{1435}, \underline{1443}, \\
\underline{1491}, \underline{1492}\end{array}$ & $\begin{array}{l}\text { A.D. } \underline{1514}, \underline{1563}, 1571 \text {, } \\
1573\end{array}$ \\
\hline Bodensee-Gfrörni & - & - & A.D. $\underline{1435}$ & A.D. $\underline{1573}$ \\
\hline Übrige Seen gefroren & - & - & - & $\begin{array}{l}\text { A.D. } 1571 \text { Comersee } \\
\text { teilweise gefroren. } \\
\text { 1573: Genfer-, Vierwald- } \\
\text { stätter- und Neuenbur- } \\
\text { gersee }\end{array}$ \\
\hline $\begin{array}{l}\text { Kältester und schneereichster } \\
\text { Winter des Jahrhunderts }\end{array}$ & - & - & - & A.D. $\underline{1565}$ \\
\hline Lawinenreichster Winter & - & - & - & - \\
\hline Gletscherausbruch & - & - & - & $\begin{array}{l}\text { A.D. } \underline{1585} \mathrm{im} \mathrm{Val} \\
\text { Tuors/Bergün }\end{array}$ \\
\hline Unfruchtbare Jahre & - & - & - & - \\
\hline Weinreben erfroren & - & A.D. $\underline{1363}$ & A.D. 1435 & - \\
\hline $\begin{array}{l}\text { Überschwemmungen, Hoch- } \\
\text { wasser }\end{array}$ & - & - & - & A.D. 1570 \\
\hline
\end{tabular}

Angaben zum Klima des 16. und 17. Jahrhunderts: Hinweise auf nasse oder kalte Sommer: A.D. 1584, $\underline{1585}, 1586, \underline{1587}, 1588,1591,1596$ (Unterstrichene Jahre stehen in Übereinstimmung mit der Naturchronik von CHR. BRÜGGER).

Hinweis 'uf Gletscherschwund: A.D. 1590 («...die alten gletscher verschmulzend vnd war in alpen grosser mangel an wasser. Es hat dieser summer etlicher mas den namhafften heissen sumer, im 1540. iar gewäsen, übertrofen ...»).

\subsection{Angaben zum Weinbau in Graubünden von F. Wassali (1859)}

Angaben zum Weinbau können bisweilen interessante Hinweise zum Klima liefern (vgl. z. B. C. PFISTER, 1981 und 1984).

Aus der "Tabelle der Weinjahre seit 1749» werden folgende schlechten Jahrgänge bzw. Fehljahre aufgeführt:

Fehljahre: $1752, \underline{1769}, 1780, \underline{1785}, \underline{1789}, 1793,1795$, $\underline{1799}, 1800,1801,1803,1805,1813,1814,1815,1816$, $1830,1850$.

Schlechte Qualität: 1760, 1764, 1775, 1809, 1812, 1817.

Geringe Qualität: $\underline{1756}, \underline{1757}, 1835,1847$.
Schwache Qualität: 1820, 1851, 1852.

(Unterstrichene Jahre stehen in Übereinstimmung mit der Naturchronik von CHR. BRÜGGER). Für das Jahr 1850 (bekanntlich ein Gletscherhochstand-Jahr) wird angegeben: spätes Frühjahr, schlechte Weinqualität, früher Frost, Schnee Ende Oktober.

\subsection{Die meteorologischen Beobachtungen von 1783-1810 in Marschlins von J.R. von Salis- Marschlins}

JOH. RUD. VON SALIS-MARSCHLINS (1756-1835), Sohn des Ministers ULYSSES VON SALIS, führte in den Jahren 1783-1810 als Edelmann und Bauer auf Schloß Marschlins bei Igis-Landquart (Graubünden) vielfältige meteorologische und phänologische Messungen bzw. Beobachtungen durch. Die Witterung wurde - so nach seinen eigenen Angaben (1804/1805, Band 1, Seiten 329-341) - im ganzen Tagesverlauf beobachtet. Es erfolgten drei Ablesungen im Tag (morgens, mittags, abends) am Barometer und am Thermometer in Grad Réaumur. Folgende Wetterelemente wurden registriert: «Stürme, Ungewitter, Thaue und Reifen» (Tab.I), ferner «Witterung und Winde nach dreimali- 
Tabelle 1b

\begin{tabular}{|c|c|c|}
\hline Aussage & 17. Jh. & 18. Jh. \\
\hline Sehr kalter/strenger Winter & - & A.D. $\underline{1708}$ \\
\hline $\begin{array}{l}\text { Strenge Winter und kühle, nasse } \\
\text { Sommer }\end{array}$ & $\begin{array}{l}\text { A.D. } \frac{1600}{161}, 1601, \\
1614,1621,1622,1625, \\
1627,1628,1634,1644,1658, \\
1664,1668, \underline{1672}, 1673, \underline{1675}, \\
1676, \underline{1677}, \underline{1680}, \underline{1684}, \frac{1685}{1683}, \\
1687, \underline{1688}, \underline{1689}, \underline{1693},\end{array}$ & 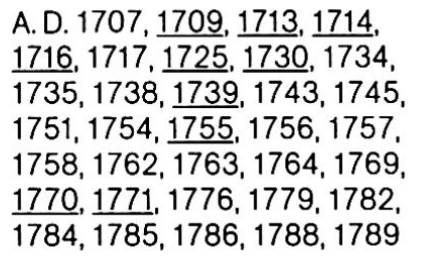 \\
\hline Zürichsee-Gfrörni & A.D. $1600,1660,1695$ & $\begin{array}{l}\text { A.D. } 1709, \frac{1716}{1717,1739}, \\
\frac{1755}{1788}, 1763,1776,1779,1784\end{array}$ \\
\hline Bodensee-Gfrörni & A.D. 1684,1695 & - \\
\hline Übrige Seen gefroren & $\begin{array}{l}\text { A. D. } 1684 \text { (Rhein), } 1695 \text { (Neuen- } \\
\text { burgersee) }\end{array}$ & $\begin{array}{l}\text { A. D. } 1755 \text { (Rhein von Chur bis } \\
\text { Basel gefroren) }\end{array}$ \\
\hline Schneereicher Winter & A.D. 1668,1689 & A.D. $\underline{1755}$ \\
\hline $\begin{array}{l}\text { Kältester und schneereichster Winter } \\
\text { des Jahrhunderts }\end{array}$ & - & A.D. 1789 \\
\hline Lawinenreicher Winter & A.D. 1689,1695 & A.D. $1720-1722,1737$ \\
\hline Gletscherausbruch & - & - \\
\hline Unfruchtbare Jahre & $\begin{array}{l}\text { A.D. } 1623,1624,1675,1688 \text {, } \\
1692\end{array}$ & A.D. $\underline{1771}$ \\
\hline Weinreben erfroren & A.D. 1675 & - \\
\hline $\begin{array}{l}\text { Gefrorene Bäume und erfrorene Men- } \\
\text { schen }\end{array}$ & A.D. 1684 & $\begin{array}{l}\text { A. D. } 1708 \text { (erfrorene Men- } \\
\text { schen in der Schweiz, in } \\
\text { Deutschland und Italien); } \underline{1709} \text {, } \\
1740,1755 \text { (viele Menschen } \\
\text { und Vieh erfroren) }\end{array}$ \\
\hline Überschwemmungen, Hochwasser & A.D. 1676 (Plurs), 1677 & $\begin{array}{l}\text { A. D. } 1705,1706,1720-1722, \\
1755,1762-\text { Jahr des Hoch- } \\
\text { wassers mit Schäden in der } \\
\text { ganzen Schweiz; } 1764: \text { Schä- } \\
\text { den besonders in Graubün- } \\
\text { den, Glarus und St. Gallen. } \\
1789 .\end{array}$ \\
\hline
\end{tabular}

gen Beobachtungen des Tages» (Tab. II). Die Tabelle III bietet eine "Zusammenstellung der barometrischen und thermometrischen Höhen").

J.R.VON SALIS-MARSCHLINS machte außerdem genaue Notizen zur Phänologie: Blühbeginn von Gehölzen, Sträuchern und Kräutern im Schloßgarten und in der nahen Umgebung; Blühbeginn der Alpenpflanzen, Kartoffeln, Reben; Ankunft der Zugvögel, erster Kuckuck-Ruf usw.; Beobachtungen zur Astronomie (Kometen), Erdbeben, später Schneefall, Spätfroste und ihre Schäden, Landwirtschaftliches, besondere Unglücksfälle, Epidemien, Mortalität der Bevölkerung. Der Schloßherr versuchte, weitere Leute zu derartigen Aufzeichungen anzuregen. So schrieb er 1804 auf Seite 346: «Es wäre mir übrigens sehr lieb, wenn sich verständige Männer in verschiedenen Gegenden unseres Vaterlandes mit ähnlichen Bemerkungen über das Klima, und über die Fruchtbarkeit der verschiedenen Jahrgänge, bemühen und mich in dieser Arbeit unterstützen wollten, die durch Vergleichung verschiedenartiger Gegenden ein größeres Interesse gewinnen würde.»

Als naß-kühlen Sommer mit schneereichem Winter bezeichnete J.R.VON SALIS-MARSCHLINS die Jahre: 1803, 1805 (auch Pfr. TRUOG von Thusis erwähnt, daß 1805 ein schlechtes, naß-kaltes Jahr gewesen sei. In: Der neue Sammler, 2 (1806): 417-421), 1808, 1809.

Folgende Weinjahrgänge in der Herrschaft Maienfeld waren Fehljahre: 1769, 1775, 1785, 1786, 1787, 1793, 1795, 1799, 1800, 1801, 1803, 1805. 


\subsection{Beiträge zur Naturchronik und Klimatologie des Ober-Engadins 1850-1900 von A. von Flugi $(1919,1920)$}

ALPHONS VON FLUGI stammte aus einem seit dem 15. Jahrhundert in St. Moritz auftretenden Geschlecht. Seit den beiden Bischöfen FLUGI im 17. Jahrhundert stand der Familie der selten benützte Titel FLUGI voN ASPERMONT zu. Die Nachkommen dieses Geschlechtes schrieben sich FLUGI, VON FLUGI oder romanisch DE FLUGI. Die Beiträge zur Naturchronik und Klimatologie des Oberengadins für 1850-1900 stammen von ALPHONS VON FLUGI aus Silvaplana. Für den ähnlichen Zeitabschnitt sammelte C.COAZ die vielfältigsten Naturereignisse, so die Witterung für jeden Monat in Chur, im Schanfigg, Oberland, Prättigau, in der Herrschaft sowie im Bergell. Sein Beobachtungsmaterial wurde im Bündner Monatsblatt veröffentlicht.

A. VON FLUGIS Angaben zur Klimatologie sind umfangreich und recht detailliert.

Eine genaue Auswertung im Sinne einer Klimareihe wäre gewiß lohnend.

Auf kühl-nasse Sommer und strenge Winter weisen folgende Jahre hin: $1850,1851,1852,1855,1866,1867$, 1871, 1872, 1875, 1879, 1890, 1891, 1919.

Das Jahr 1862 wies für 1850-1900 im Oberengadin die höchsten Monatsmittel der Temperatur auf. 1865 wurde der St. Moritzersee für die Periode 1850-1900 am frühesten eisfrei, und zwar am 28. A pril.

Als Jahre mit ungewöhnlich spätem Frühling nennt A. VON FLUGI: 1817, 1829, 1836, 1837, 1843, 1855, 1879, 1901, 1902, 1910, 1916, 1917.

Ungewöhnliche Kälteperioden herrschten 1891, 1901, 1905, 1915, 1919.

Hinweise auf schneereiche Winter: $1850,1851,1855$, $1867,1871,1872$ (dieses war das niederschlags- und schneereichste Jahr von 1864-1900), 1875, 1879, 1886, 1888 (ausgesprochen viel Schnee, häufig Lawinen und Hochwasser), 1890.

\subsection{Schneelawinen in St. Anthönien (Prättigau) von 1690-1804 nach Aufzeichnungen von Catani und Engel (1805)}

CATANI und ENGEL (1805) haben in ihrer "Beschreibung des Thals St. Anthönien im Brättigau» die Schneelawinen von 1690-1804 und die damit verbundenen Todesopfer aufgezeichnet:

Jahr Anzahl Lawinentote

\begin{tabular}{lc}
\hline $1689 / 1690$ & 13 \\
1714 & 1 \\
1720 & 4 \\
1731 & 6 \\
1737 & 2 \\
1756 & 3 \\
1776 & 4 \\
1797 & 1 \\
& Total: 34
\end{tabular}

\section{8 "Das Clima der Alpen ...", ein Aufsatz von Pater Placidus a Spescha (1818)}

Der Disentiser Mönch und Pater zu Truns, PLACIDUS a SPESCHA (1752-1833), verfaßte 1818 diesen Aufsatz «Das Clima der Alpen, am Ende des vorigen und im Anfange des jetzigen Jahrhunderts» als Anlaß zu der damals von der Schweizerischen Naturforschenden Gesellschaft gestellten Frage, ob das Klima in den Alpen in den letzten Jahren wirklich rauher geworden sei. Das zunächst unveröffentlicht gebliebene Manuskript wurde 1868/1869 vom Bündner Naturforscher G. THEOBALD im SAC-Jahrbuch, Band 5, herausgegeben und kommentiert.

P. a SPESCHA führte von 1783-1818 Beobachtungen zum Klima Graubündens durch. Besonders seit 1811 sei es merklich rauher geworden. Als diesbezügliche Hinweise nennt P. a SPESCHA: 1. Die Alpweiden sind mit Schnee und Eis bedeckt. 2. Verminderter Holzwuchs. 3. Zunahme der Gletscher.

P. a SPESCHA erwähnt besonders die veränderten Verhältnisse der verschiedenen Berggipfel und Gletscher des Vorder- und Hinterrheintals, die er früher bestiegen hatte. Als Ursachen der Klimaverschlechterung nennt der Autor:

Primär: Meeresregression und das damit verbundene Aufsteigen des Festlandes. Sekundär: Verstärkte Anhäufung von Schnee und Eis in den Alpen. G.THEOBALD kommentiert diese Begründungen im Lichte der damaligen naturwissenschaftlichen $\mathrm{Be}$ trachtungsweise, der P. a SPESCHA behaftet war. SPESCHAS Einzelbeobachtungen sind nicht weiter tabellarisch auszuwerten. Über diesen sehr vielseitigen Bündner Geistlichen schrieb der Disentiser Pater Dr. I. MÜLLER (1974) eine wertvolle, gut dokumentierte Biographie.

\section{Synthese und Vergleich mit Gletscherschwankungen in Graubünden}

Die BRÜGGERsche Naturchronik enthält eine große Fülle verschiedenster Informationen, die in Kap. 2 bezüglich der für die paläoklimatische Forschung festgesetzten Stichworte ausgewertet wurde. Für das Mittelalter konnten eine ganze Reihe von Jahren mit strengen Wintern und kühlen, nassen Sommern notiert werden: Im 11. Jahrhundert sind es besonders die vierziger und fünfziger Jahre; im 13. Jahrhundert sind es die zwanziger und dreißiger Jahre. Im 14. Jahrhundert ragt das Jahr 1363 hervor durch strengen Winter, Zürichsee-Gfrörni und Erfrieren der Weinreben. Das 15. Jahrhundert ist besonders in den dreißiger und vierziger Jahren durch Klimaungunst gekennzeichnet. Im 16. Jahrhundert herrschten in den zehner, sechziger, siebziger, achtziger und neunziger Jahren kühle, nasse Sommer und strenge Winter mit weitverbreiteter Seegfrörni: Zürich-, Boden-, Genfer-, Vierwaldstätter- und Neuenburgersee und sogar teilweise der Comersee. Im Jahr 1585 erfolgte im Val 
Tuors/Bergün ein «Gletscherausbruch». Das Jahr 1570 ist reich an Überschwemmungen und Hochwasserkatastrophen gewesen. Das 16. Jahrhundert muß mit Ausnahme der zwanziger bis fünfziger Jahre klimatisch besonders unwirtlich gewesen sein. (Oder täuscht dies die größere Informations-Fülle lediglich vor?)

Eine besonders gute Übereinstimmung der Ereignisse ergibt sich im Vergleich mit H.ARDÜSERS Chronik. Möglicherweise diente diese CHR. BRÜGGER als Quelle. Die kulturgeschichtlich sehr interessanten Aufzeichnungen H. ARDÜSERS von 1572-1614 lieferten jedoch für klimatologische Fragestellungen eine sehr bescheidene Ausbeute. Die Angaben in der Naturchronik von E. KILLIAS, welche mit dem Jahr 1866 beginnen, bilden in gewissem Sinne die Fortsetzung der BRÜGGERschen Aufzeichnungen, die im Jahr 1800 enden.

Eine klimageschichtlich ergiebige Quelle stellt die "Tabelle der Weinjahre seit 1749»" zum Weinbau Graubündens von F. WASSALI (1859) dar. Hier können besonders gute Koinzidenzen mit den neuzeitlichen Gletscherbewegungen festgestellt werden.

Die wenigen klaren Angaben J.R. von SALIS' zu naßkalten Sommern und schneereichen Wintern im ersten Jahrzehnt des 19. Jahrhunderts stimmen vor allem überein mit den mißlungenen Weinjahrgängen. Weitgehende Übereinstimmung liegt auch vor im Vergleich mit den Angaben F. WASSALIS und den Vorstößen der Bernina-Gletscher (F. BEELER, 1977).

Das Datenmaterial A. voN FLUGIS (1919/1920) über naß-kalte Sommer und strenge bzw. schneereiche Winter oder späten Frühlingsbeginn umfaßt die Zeit von der Mitte des 19. Jahrhunderts bis zum zweiten Jahrzehnt des 20. Jahrhunderts.

Besonders gute Koinzidenzen mit den Angaben F. WASSALIS, E.KILLIAS' und F. BEELERS liegen für die fünfziger Jahre des 19. Jahrhunderts vor. Eine weitere gute Übereinstimmung zeigen die siebziger und achtziger Jahre des vorigen Jahrhunderts. Die lawinenreichen Jahre des 17. und 18. Jahrhunderts in St. Anthönien, gesammelt von CATANI und ENGEL (1805), stehen wiederum in engem Zusammenhang mit den mißlungenen Wein-Jahrgängen (vgl. die Tab. 2).

Die Tabelle 2 stellt die oben genannten Informationen aus den Bündner Naturchroniken einigen (publizierten) Vorstößen bzw. Hochständen von Gletschern Graubündens gegenüber. Hierzu wurden folgende Publikationen ausgewählt: F. BEELER (1977), C. BURGA (1981), M. MALISCH (1981) und J.SUTER (1981). Besonders die Angaben F. BEELERS zu den Gletschern des Bernina-Gebietes erlauben aufschlußreiche Vergleiche mit den Daten der BRÜGGERschen Naturchronik. Man gewinnt den Eindruck, daß die strengen Winter und kühlen, nassen Sommer der Jahre 1770/71, 1782-1789 und 1799 in direktem kausalen Zusammenhang mit den Ausdehnungen der Palü-, Cambrena- und Morteratsch-Gletscher um 1780, 1793 und 1820 stehen dürften, wobei die Eisströme mit einer gewissen Trägheit gegenüber dem Klimageschehen reagiert haben.

Für die von F. BEELER im 17. Jahrhundert festgestellten Oszillationen des Palü- und Cambrena-Gletschers finden sich zahlreiche Hinweise zu Klimaverschlechterungen in der BRÜGGERschen Chronik, so mit kurzen Unterbrüchen über 20 Jahre hinweg von 1672 bis 1695 .

Die Daten aus den Aufzeichnungen von E. KILlias und besonders von A. VON FLUGI lassen ebenfalls einen deutlichen Zusammenhang mit den Gletscherbewegungen aus dem Oberengadin, dem Albula- und dem Hinterrheintal feststellen. Besonders hervorzuheben sind wiederum die Gletscher der Bernina-Gruppe und ferner der Val Bever.

Wie bereits oben erwähnt, scheinen die Fehljahre des Bündner Weinbaues (F. WASSALI, 1859; J.R. VON SALIS, 1783-1810) einen besonders engen Konnex mit den Gletscherschwankungen des 18. und 19. Jahrhunderts aufzuweisen (vgl. Tab. 2).

Als Beispiele seien die Jahre 1780, 1793, 1820 und 1850/51 genannt, wo die Fehljahre bzw. schlechte Weinqualität in direktem Zusammenhang mit den entsprechenden Gletschervorstößen bzw. -hochständen stehen.

Ein Vergleich des hier verarbeiteten Datenmaterials aus Graubünden mit den von H. VON RUDLOFF (1980) und C. PFISTER (1984) herausgearbeiteten Grundzügen zum Klimaverlauf Mitteleuropas seit dem 16. Jahrhundert zeigt weitgehende Übereinstimmung. Die Klimaverschlechterung von 1565-1629 ist mehrfach durch das Quellenmaterial CHR. BRÜGGERS (1882/1888) und durch das Tagebuch H.ARDÜSERS (1572-1614) belegt.

Zum Höhepunkt der «Kleinen Eiszeit» von 1688-1701 nennt CHR. BRÜGGER mehrere Jahrgänge mit strengem Winter, naß-kühlem Sommer und des öftern Seegfrörni.

Die «kontinentalen Tendenzen» (C. PFISTER, 1984) der Jahre 1731-1811 scheinen ihren Niederschlag in einer Serie von Wein-Fehljahren zu finden (vgl. F. WASSALI, 1859). Es sind dies die Jahrgänge der fünfziger, achtziger und neunziger Jahre. J.R. VON SALIS (1783-1810) nennt für diesen Abschnitt ebenfalls eine ganze Reihe von Wein-Fehljahren.

Die Kaltperiode von 1812-1860 findet mehrfache Bestätigung im reichen klimatischen Datenmaterial A. vON FLUGIS (1919/1920) für das Oberengadin (vgl. Tab. 2). Es sei lediglich noch daran erinnert, daß 1812 der russische Feldzug Napoleons I. stattfand, welcher bekanntlich mit einer völligen Katastrophe endete, nicht zuletzt auch wegen des russischen Winters.

H. VON RUDLOFF (1980) erwähnt, daß in den Jahren 1817-1848 Massenauswanderungen von deutschen Weinbauern u.a. nach Amerika stattfanden. Für Graubünden ist ebenfalls in dieser Periode eine massive Auswanderungswelle zu konstatieren. Hier waren es hauptsächlich Bauern und Handwerker, die wegen der drückenden Armut nach Amerika und 


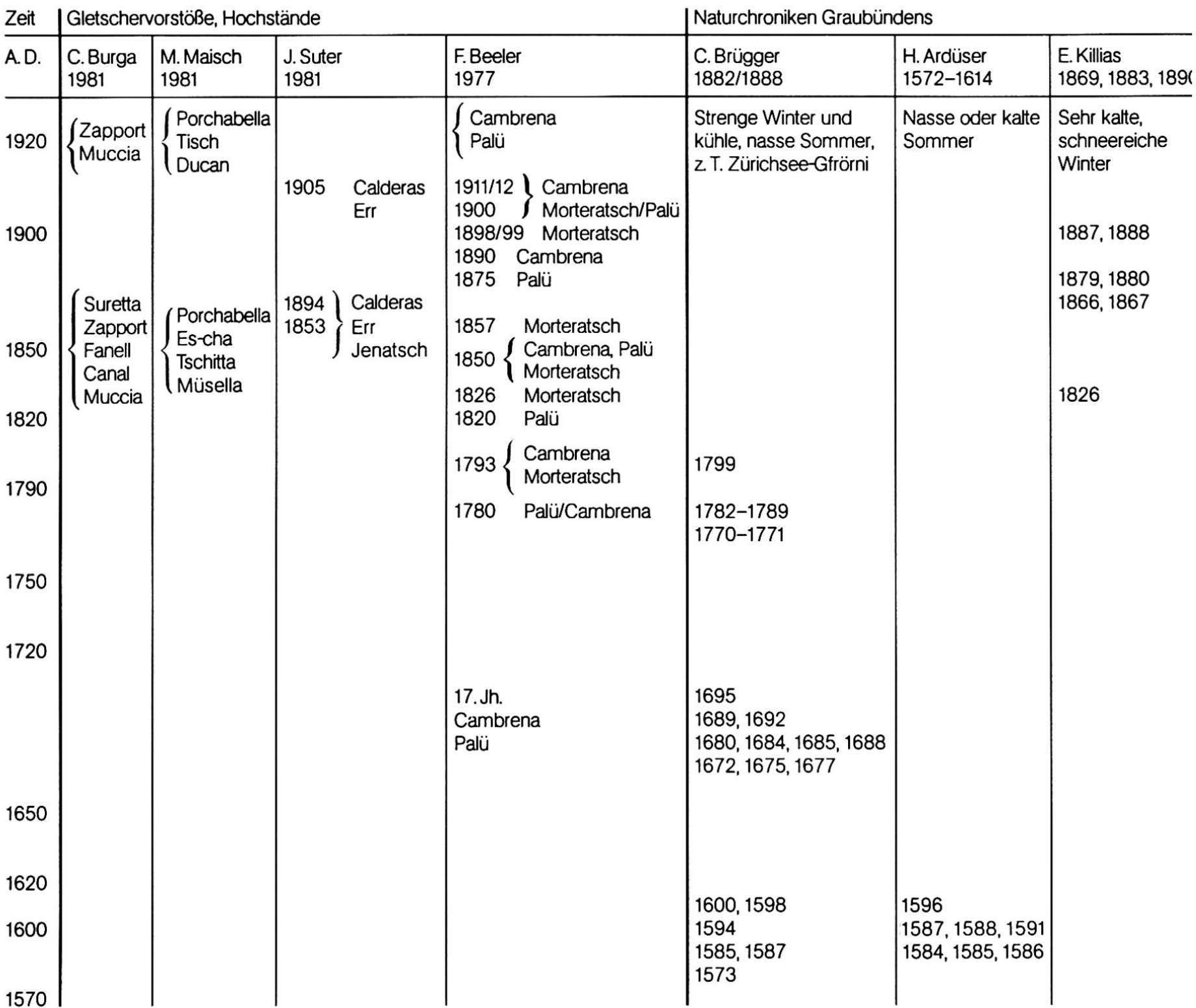

Australien auswanderten, um dort als Farmer und Goldgräber ihr Glück zu suchen.

Die nach H.FLOHN und M. RODEWALD (1975, zit. in H. VON RUDLOFF, 1980) "stabile Klimaperiode» von 1897-1939 wurde aber von mehreren beträchtlichen Gletschervorstößen um 1915-1926 unterbrochen.

\section{Zusammenfassung}

Das Beobachtungsmaterial über Naturerscheinungen Graubündens kann in fünf Gruppen eingeteilt werden: 1. Beobachtungen von einheimischen Intellek- tuellen, 2. Alte Chroniken, 3. Naturchroniken (Aufzeichnungen verschiedenster Naturerscheinungen), 4. Berichte von Forschungsreisenden, 5. Briefe und Berichte der übrigen Reisenden.

Das sehr unterschiedliche Datenmaterial über Graubünden ist vorwiegend qualitativer Natur. Die zeitlich am weitesten bis ins 11. Jahrhundert zurückreichende Bündner Naturchronik verfaßte CHR. BRÜGGER (1882/1888). Nebst diesen Aufzeichnungen wurden folgende Quellen ausgewertet: Naturchronik von E. KILLIAS für die Jahre 1867, 1880 und 1888; Chronik von H. ARDÜSER (1572-1614); Angaben zum Weinbau in Graubünden von F.WASSALI (1859); meteorolo- 


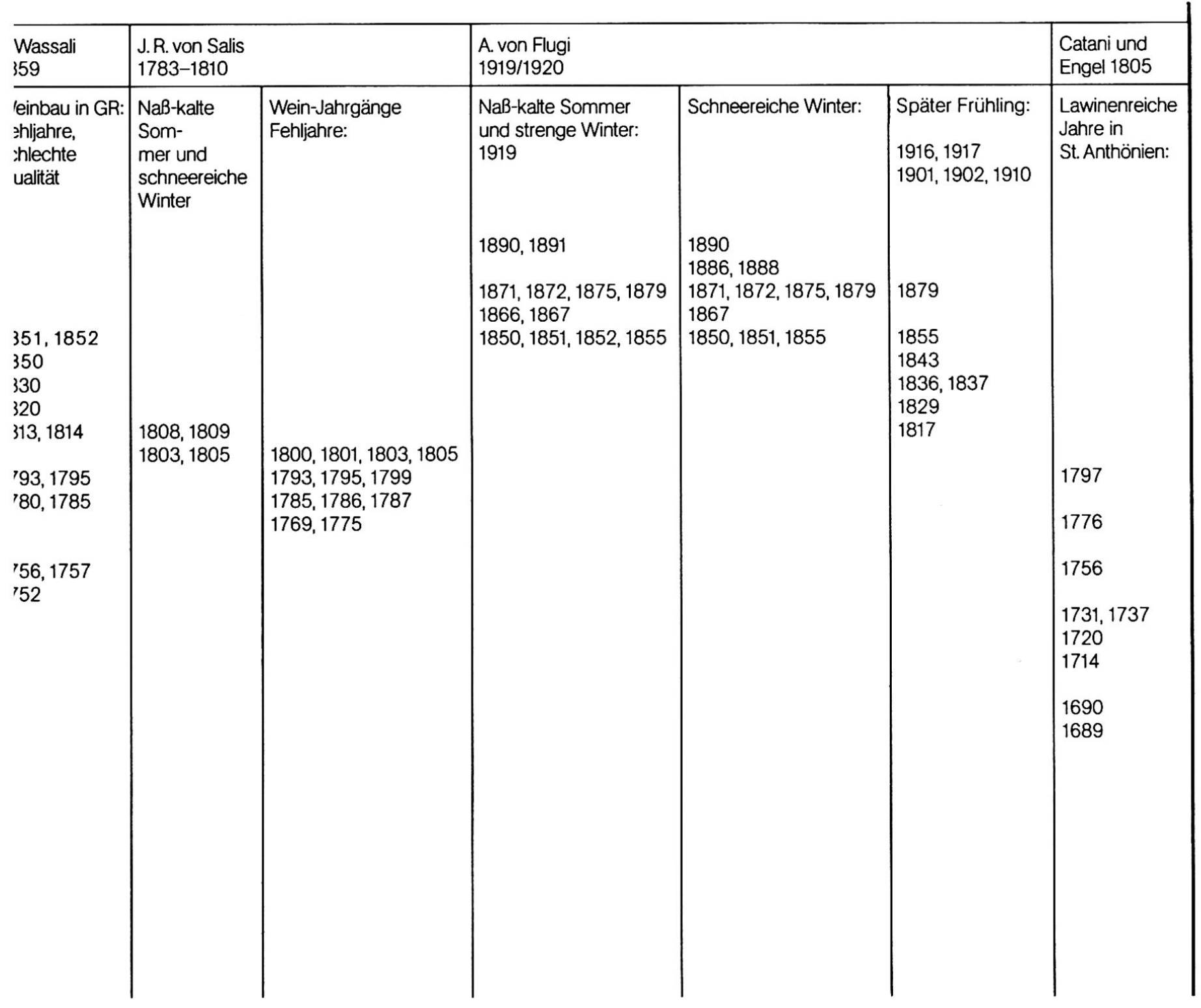

gische Beobachtungen von 1783-1810 in Marschlins (Landquart) von J.R. vON SALIS-MARSCHLINS; Naturchronik und Klimatologie des Oberengadins von 1850 bis 1900 von A. VON FLUGI (1919/1920); Schneelawinen in St. Anthönien von 1690-1804 nach Aufzeichnungen von CATANI und ENGEL (1805); «Das Clima der Alpen...» von Pater P. a SPESCHA.

Die paläoklimatischen Informationen aus den oben genannten Quellen werden einerseits gegenseitig und anderseits mit den Gletschervorstößen bzw. -hochständen einiger Bündner Täler verglichen (Hinterrheintal, Albula, Oberengadin, Bernina). Die Naturchronik CHR. BRÜGGERS liefert für das 17.-20. Jahrhun- dert diesbezüglich zahlreiche Übereinstimmungen (vgl. Tab.2). Besonders auffällig stimmen die Fehljahre des Weinbaues mit den Gletscherbewegungen des 18. und 19. Jahrhunderts überein.

Gute Übereinstimmungen mit den Angaben F.WASSALIS, E. KILLIAS' und F. BEELERS liegen für die fünfziger, siebziger und achtziger Jahre des 19. Jahrhunderts vor. Die von F.BEELER für das 17. Jahrhundert festgestellten Oszillationen des Palü- und Cambrenagletschers finden zahlreiche Bestätigungen in der BRÜGGERschen Chronik. Die Aufzeichnungen A. vON FLUGIS und E. KILLIAS' lassen deutliche Zusammenhänge mit den Gletscherbewegungen aus dem Ober- 
engadin, dem Albula- und dem Hinterrheintal erkennen.

Eine Gegenüberstellung des hier verarbeiteten Informationsmaterials aus Graubünden mit den von H. vON
RUDLOFF (1980) und C. PFISTER (1984) herausgearbeiteten Grundzügen zum Klimaverlauf Mitteleuropas seit dem 16. Jahrhundert zeigt weitgehende Übereinstimmung.

\section{Literaturverzeichnis}

ALEXANDRE P. (1977): Le climat dans l'histoire au moyen âge In: Annales, Rev. bimestrielle sur Economies, Sociétés, Civilisations, Paris: 183-197.

ARDÜSER H. (1572-1614): Chronik. Hrsg. von J.BOTT (1877): Hans Ardüsers rätische Chronik. Commentar. Jb. Natf. Ges Graubünden, N. F.: 43-638.

BEELER F. (1977): Geomorphologische Untersuchungen am Spät- und Postglazial im Schweizerischen Nationalpark und im Berninapaßgebiet (Südrätische Alpen). Erg. wiss. Unters. Schweiz. Nationalpark, 15, 77: 131-276.

BRÜGGER CHR. (1882/1888): Naturchronik der Schweiz insbesondere der Rhätischen Alpen. Programm der Bündnerischen Kantonsschule Chur, I.-V.Folge (1882), VI. Folge (1888).

BRUNNER H. (1970): Chr. Georg Brügger (1833-1899). In Bedeutende Bündner, 2: 140-148.

BUNDI M. (1982): Zur Besiedlungs- und Wirtschaftsgeschichte Graubündens im Mittelalter. Chur, $693 \mathrm{~S}$

BURGA C.A. (1981): Glazialmorphologische Untersuchungen im Hinterrheintal und am Bernhardin-Paß. Vjschr. Natf. Ges. in Zürich, 126, 4: 237-267.

CATANI und ENGEL (1805): Beschreibung des Thals St. Anthönien im Brättigau. Der neue Sammler 1: 465-488.

FLUGI A. von (1910/1920): Beiträge zur Naturchronik und Klimatologie des Ober-Engadins 1850-1900. In: Bündner Monatsblatt: $222-224, \quad 249-254, \quad 280-290, \quad 349-351$ 380-383 bzw. 23-27, 50-54, 100-106, 115-118, 308-312, $328-335$

KILLIAS E. (1869/1883/1890): Periodische Erscheinungen und Naturereignisse. Jber. Natf. Ges. Graubünden, 14, 26, 33: 184-188, 33-37, 67-71.

MAISCH M. (1981): Glazialmorphologische und gletschergeschichtliche Untersuchungen im Gebiet zwischen Landwasserund Albulatal (Kt. Graubünden, Schweiz). Phys. Geogr., 3, $215 \mathrm{~S}$.

MARGADANT S. (1978): Land und Leute Graubündens im Spiegel der Reiseliteratur 1492-1800. Zürich, 282 S.

MARGADANT S. (1980): "Der Sammler" und "Der neue Sammler.» In: Bündner Monatsblatt, 1-2: 1-22.

MOOR C. von (1853): Fortunat von Juvaltas Denkwürdigkeiten. Ulrich Campells zwei Bücher rätischer Geschichte. In: Archiv für die Geschichte der Republik Graubünden. Chur, 1: 1-108, 1-236 und 2: 1-568.

MOOR C. von (1876): Geschichte von Currätien und der Republik gemeiner drei Bünde, 3 Bände.

MÜLLER I. (1971): Glanz des rätischen Mittelalters. KristallReihe, 6, Chur, $111 \mathrm{~S}$.

MÜLLER I. (1974): Pater Placidus Spescha 1752-1833. Disentis, $174 \mathrm{~S}$.
MÜLLER P.E. (1972): Dichter erleben Graubünden. KristallReihe, 7, Chur, $111 \mathrm{~S}$.

OESCHGER H., MESSERLI B. und SVILAR M. (1980): Das Klima Berlin, $296 \mathrm{~S}$.

PFISTER C. (1977): Zum Klima des Raumes Zürich im späten 17. und frühen 18. Jahrhundert. Vjschr. Natf. Ges. in Zürich, 122, 4: 447-471.

PFISTER C. (1981): Die Fluktuationen der Weinmosterträge im Schweizerischen Weinland vom 16. bis ins frühe 19. Jahrhundert. Klimatische Ursachen und sozio-ökonomische Bedeutung. In: Schweiz. Zt. f. Geschichte, 31/4: 445-491.

PFISTER G. (1984): Klimageschichte der Schweiz 1525-1860. 2 Bände, Bern und Stuttgart, Academica helvetica, 6.1 und 6.2, 184 und $163 \mathrm{~S}$.

PIETH F. (1982): Bündnergeschichte. Nachdruck, Chur, 638 S.

PLANTA J.M. von (1970): Conradin von Flugi 1787-1874 und Alfons von Flugi 1823-1890. In: Bedeutende Bündner aus fünf Jahrhunderten, 1: 473-485.

RIBI H. (1973): Schreckliche Viamala. Kristall-Reihe, 11, Chur, $80 \mathrm{~S}$

RUDLOFF H. von (1967): Die Schwankungen und Pendelungen des Klimas in Europa seit dem Beginn der regelmäßigen Instrumenten-Beobachtungen (1670). Die Wissenschaft, 122 Braunschweig.

RUDLOFF H. von (1980): Die Klima-Entwicklung in den letzten Jahrhunderten im mitteleuropäischen Raume (mit einem Rückblick auf die postglaziale Periode). In: H. Oeschger, B. Messerli und M. Svilar, 1980: Das Klima: 125-148.

SALIS J.R. von (1804-1812): Meteorologische Beobachtungen der Jahre 1802-1808. Der neue Sammler, 1: 329-341; 2 : $68-77,338-350,403-417 ; 3$ : 269-280; 4: 356-373; 5: 151-168; 7: 165-183.

SALIS J.U. von (1811): Einige Resultate aus sechs und zwanzigjährigen Witterungsbeobachtungen in Marschlins. Der neue Sammler, 6: 193-211.

SALIS-SOGLIO N. von (1891): Die Familie von Salis. Lindau, $368 \mathrm{~S}$.

SIMONETT C. (1970): Hans Ardüser (1557-1618). In: Bedeutende Bündner aus fünf Jahrhunderten, 1: 127-128.

SPESCHA P. a (1818): Das Clima der Alpen, am Ende des vorigen und im Anfange des jetzigen Jahrhunderts. SAC-Jb., 5 (1868/1869): 494-501. Hrsg. von G. Theobald.

SUTER J. (1981): Gletschergeschichte des Oberengadins: Untersuchung von Gletscherschwankungen in der Err-JulierGruppe. Phys. Geogr., 2, 147 S.

TRUOG Pfr. (1806): Sommer- und Herbstwitterung im Jahr 1805. Der neue Sammler, 2: 417-421.

WASSALI F. (1859): Der Weinbau im Kanton Graubünden. In: Jber. Natf. Ges. Graubünden, 4: 59-76. 